\title{
Data Models and Digital Twins for the Digital Economy: Real, Augmented, and Synthetic Data in Decision Support Systems
}

\section{Minitrack Introduction}

Digital transformation is an ongoing, long-term and disruptive change process based on the continuous progress of information technology. It is reasonable to assume that the advent of digital transformation is changing the way the economy works on a macro and micro level. Therefore, the theory and practice of economics needs to shift to digital economics with a strong emphasis on the impact of information technologies on eco-systems, enterprises, households and other relevant stakeholder groups.

An important approach to research on digital economics is related to models and data. Relevant research problems in the real world encompass a large number of variables, dynamic interdependences, high complexity, ambiguity, and multiple options. Therefore, models are used to understand real-world processes and to explore the impact of decision options. Usually, such models are linked to or tested with empirical data. Approaches for data modeling cover probabilistic and deterministic approaches from different sub-fields, such as econometrics, machine learning, structural equation modeling and system dynamics. Such data models are often used as digital twins for real-life problems and deployed as a decision support system.

An important limitation of the application of data models and digital twins is the absence, incompleteness or reliability of data. Therefore, real-world data must be captured and approved by multiple procedures (e.g. surveys, census, sensors). Real-world data may also be complemented by augmented data or substituted by synthetic data. This leads to the notion of augmented or synthetic data twins and the possibility of rapid prototyping, simulations and shorter time-to-market cycles for model-based decision support systems.

The HICSS minitrack "Data Models and Digital Twins for the Digital Economy: Real, Augmented, and Synthetic Data in Decision Support Systems" aims to explore these issues, paying particular attention to the challenges of data model development, data model evaluation, augmented or synthetic data models and digital twins.
Finally, two papers were accepted to be included in this minitrack. The paper of Barth et al. works on "Value Generation in the Product Lifecycle with Digital Twins: Status Quo in Swiss Companies". Value generation with digital twins along the phases of the product lifecycle affects different processes from a company's internal perspective. The study of Barth et al. investigated in which phases of the product lifecycle and processes swiss companies seek to generate what value contribution. The results of the survey of 103 companies show that only $47 \%$ of the companies surveyed are applying or planning to apply digital twins. The motivation primarily lies in increasing efficiency (79\%) or in building competitive advantage (63\%). These companies clearly recognize the potential of digital twins to create value in the Beginning-of-Life-phase (79\%) and in the Middle-ofLife-phase (60\%). In contrast, the use in the End-ofLife-phase (15\%) is subordinate. The companies seek to generate value with digital twins by offering qualitatively better products and services in a shorter time or with higher availability. Cost savings seem secondary and overestimated by companies before they apply digital twins.

The second paper in the minitrack by Rossmann and Hertweck is titled "Digital Twins: A Meta-Review on Their Conceptualization, Application, and Reference Architecture". The concept of digital twins is receiving increasing attention in research and management practice. However, various facets around the concept are blurry, including conceptualization, application areas, and reference architectures for digital twins. A review of preliminary results regarding the emerging research output on digital twins is required to promote further research and implementation in organizations. To do so, this paper asks four research questions: (1) How is the concept of digital twins defined? (2) Which application areas are relevant for the implementation of digital twins? (3) How is a reference architecture for digital twins conceptualized? and (4) Which directions are relevant for further research on digital twins? With regard to research methods, Rossmann and Hertweck conducted a meta-review of 14 systematic literature reviews on digital twins. The results yield important insights for the current state of research on digital twins. 
Application areas for digital twins are expanding and cover a broad array of domains, from manufacturing and healthcare to smart cities, logistics, business, economics, and even psychology. However, a common reference architecture for digital twins can define the relevant properties of digital twins over several domains. Therefore, the paper of Rossmann and Hertweck presents a unified reference architecture with nine distinct layers as a blueprint for the configuration of digital twins. Finally, the meta-review unpacks future research directions in eight different areas: concept development, business model, integration, data entry, big data, data analysis, standardization, and security and privacy. This opens pathways for future research and highlights the challenges for the further practical implementation of digital twins. 